\title{
SUPPORTING INFORMATION \\ Comparison between Different Hybrid Life Cycle Assessment Methodologies: A Review and Case Study of Biomass-based p-Xylene Production
}

\author{
Yuqing Luo ${ }^{1}$, Marianthi Ierapetritou ${ }^{1 *}$ \\ 1. University of Delaware, Department of Chemical and Biomolecular Engineering, 150 Academy \\ Street, Newark, DE 19716 \\ *Corresponding Author: mgi@udel.edu
}

\section{SI Contents:}

1) Process LCA inventory for the MSH and CA processes

2) Foreground processes of MSH process in the technological matrix

3) Summary of unit prices

4) $\mathrm{CO}_{2}$ Emission During Natural Gas Combustion

5) Technoeconomic Analysis Results Used in the Tiered Hybrid LCA 


\section{1) Process LCA Inventory}

Table S1. MSH process inventory before allocation.

\begin{tabular}{|c|c|c|c|}
\hline Inputs & Unit & Quantity & Ecoinvent names \\
\hline Biomass & metric ton & 50 & $\begin{array}{l}\text { Wood chips, wet, measured as dry mass }\{\mathrm{DE}\} \mid \text { hardwood forestry, oak, sustainable forest } \\
\text { management } \mid \text { Alloc Def, } U\end{array}$ \\
\hline $\mathrm{H} 2 \mathrm{SO} 4$ & metric ton & 0.004 & Sulfuric acid $\{$ RoW $\} \mid$ production $\mid$ Alloc Def, $U$ \\
\hline Water & metric ton & 562.4 & Water, deionised, from tap water, at user $\{\mathrm{RoW}\} \mid$ production $\mid$ Alloc Def, $U$ \\
\hline $\mathrm{LiBr}$ & metric ton & 1.769 & Stoichiometric ratio of $\mathrm{LiOH}$ and $\mathrm{HBr}$ to surrogate \\
\hline Ethyl Acetate & metric ton & 0.016 & Ethyl acetate $\{$ RoW $\} \mid$ production $\mid$ Alloc Def, $U$ \\
\hline $\mathrm{AlCl} 3$ & metric ton & 0.171 & Aluminum chloride $\{\mathrm{GLO}\} \mid$ aluminum chloride production $\mid$ APOS, $\mathrm{U}$ \\
\hline $\mathrm{H} 2$ & metric ton & 0.601 & Hydrogen, liquid $\{$ RoW $\} \mid$ market for $\mid$ Alloc Def, $U$ \\
\hline Heptane & metric ton & 0.092 & Solvent, organic $\{\mathrm{GLO}\} \mid$ heptane to generic market for $\mid$ Alloc Def, $\mathrm{U}$ \\
\hline Ethylene & metric ton & 2.805 & Ethylene, average $\{R E R\} \mid$ production $\mid$ Alloc Def, $U$ \\
\hline transport & $\mathrm{tkm}$ & 5000 & Transport, freight, lorry 16-32 metric ton, EURO4 \{GLO $\} \mid$ market for $\mid$ Alloc Def, $U$ \\
\hline Hot Oil & GJ & 11.29 & $\begin{array}{l}\text { Heat, central or small-scale, natural gas }\{\mathrm{RoW}\} \mid \text { heat production, natural gas, at boiler } \\
\text { modulating }<100 \mathrm{~kW} \mid \text { Alloc Def, } \mathrm{U}\end{array}$ \\
\hline MP Steam & GJ & 91.03 & Heat, in chemical industry $\{$ RoW $\} \mid$ market for $\mid$ Alloc Def, $U$ \\
\hline Outputs & Unit & Quantity & Ecoinvent names \\
\hline Furfural & metric ton & 8.227 & \\
\hline p-Xylene & metric ton & 10.16 & \\
\hline $\begin{array}{l}\text { Electricity } \\
\text { (excess) }\end{array}$ & GJ & 55.09 & "Avoided product", Electricity, medium voltage $\{\mathrm{US}\} \mid$ market group for $\mid$ Alloc Def, U \\
\hline Wastewater & metric ton & 11.92 & $\begin{array}{l}\text { Wastewater, average }\{\mathrm{CA}-\mathrm{QC}\} \mid \text { treatment of wastewater, average, capacity } 1 \mathrm{E} 91 / \text { year } \\
\text { Alloc Def, } U\end{array}$ \\
\hline $\mathrm{CO} 2$ & metric ton & 47.06 & $\mathrm{CO} 2$ emitted to air \\
\hline
\end{tabular}

Table S2. CA process inventory before allocation.

\begin{tabular}{llrl}
\hline Inputs & Unit & Quantity & Ecoinvent names \\
Biomass & metric ton & 50 & $\begin{array}{l}\text { Wood chips, wet, measured as dry mass }\{D E\} \mid \text { hardwood forestry, oak, sustainable forest } \\
\text { management } \mid \text { Alloc Def, } U\end{array}$ \\
DME & metric ton & 0.714 & Dimethyl ether $\{$ RoW $\} \mid$ production $\mid$ Alloc Def, $U$ \\
H3PO4 & 0.375 & $\begin{array}{l}\text { Phosphoric acid, industrial grade, without water, in } 85 \% \text { solution state }\{\text { RoW }\} \mid \text { purification of } \\
\text { wet-process phosphoric acid to industrial grade, product in } 85 \% \text { solution state } \mid \text { Alloc Def, } U\end{array}$ \\
H2SO4 & metric ton & 0.751 & Sulfuric acid $\{$ RoW $\} \mid$ production $\mid$ Alloc Def, $U$ \\
Lime & metric ton & 1 & Lime $\{C A-Q C\} \mid$ lime production, milled, loose $\mid$ Alloc Def, $U$ \\
water & metric ton & 479.6 & Water, deionised, from tap water, at user $\{$ RoW $\} \mid$ production $\mid$ Alloc Def, $U$ \\
Ethyl Acetate & metric ton & 0.07 & Ethyl acetate $\{$ RoW $\} \mid$ production $\mid$ Alloc Def, U \\
AlCl3 & metric ton & 0.953 & Aluminum chloride $\{$ GLO $\} \mid$ aluminum chloride production $\mid$ APOS, $U$
\end{tabular}




\begin{tabular}{|c|c|c|c|}
\hline $\mathrm{H} 2$ & metric ton & 0.507 & Hydrogen, liquid $\{$ RoW $\} \mid$ market for $\mid$ Alloc Def, $U$ \\
\hline Heptane & metric ton & 0.597 & Solvent, organic $\{\mathrm{GLO}\} \mid$ heptane to generic market for $\mid$ Alloc Def, $U$ \\
\hline Ethylene & metric ton & 2.805 & Ethylene, average $\{R E R\} \mid$ production $\mid$ Alloc Def, $U$ \\
\hline transport & tkm & 5000 & Transport, freight, lorry 16-32 metric ton, EURO4 $\{\mathrm{GLO}\} \mid$ market for $\mid$ Alloc Def, U \\
\hline Electricity & GJ & 9.493 & Electricity, medium voltage $\{U S\} \mid$ market group for $\mid$ Alloc Def, $U$ \\
\hline Hot Oil & GJ & 10 & $\begin{array}{l}\text { Heat, central or small-scale, natural gas }\{\text { RoW }\} \mid \text { heat production, natural gas, at boiler } \\
\text { modulating }<100 \mathrm{~kW} \mid \text { Alloc Def, } U\end{array}$ \\
\hline $\begin{array}{l}\text { Refrigerant } \\
\text { Cooling }\end{array}$ & GJ & 40 & Cooling energy $\{\mathrm{GLO}\} \mid$ market for $\mid$ Alloc Def, $U$ \\
\hline Outputs & Unit & Quantity & Ecoinvent names \\
\hline Furfural & metric ton & 7.227 & \\
\hline p-Xylene & metric ton & 8.918 & \\
\hline Gypsum waste & metric ton & 2.82 & Waste gypsum $\{\mathrm{CH}\} \mid$ treatment of, inert material landfill $\mid$ Alloc Def, $U$ \\
\hline Wastewater & metric ton & 121.9 & $\begin{array}{l}\text { Wastewater, average }\{\mathrm{CA}-\mathrm{Q} C\} \mid \text { treatment of wastewater, average, capacity } 1 \mathrm{E} 91 / \text { year } \mid \text { Alloc } \\
\text { Def, } U\end{array}$ \\
\hline $\mathrm{CO} 2$ & metric ton & 46.97 & $\mathrm{CO} 2$ emitted to air \\
\hline
\end{tabular}

\section{2) Foreground Processes in the Technological Matrix}

Table S3. The four foreground processes of MSH process in the technological matrix

\begin{tabular}{|l|rrrr|}
\hline & furfural & \multicolumn{1}{l}{$\begin{array}{l}\text { p-Xylene } \\
\text { (metric ton) }\end{array}$} & LiBr & \multicolumn{2}{l|}{$\mathrm{HBr}$} \\
& (metric ton) & (metric ton) & (metric ton) \\
\hline Furfural (metric ton) & 0 & 0 & 0 & 0 \\
$p$-Xylene (metric ton) & 0 & 0 & 0 & 0 \\
LiBr (metric ton) & 0.096 & 0.096 & 0 & 0 \\
HBr (metric ton) & 0 & 0 & 0.931 & 0 \\
\hline H2S (metric ton) & 0 & 0 & 0 & 0.21 \\
Br2 (metric ton) & 0 & 0 & 0 & 0.988 \\
LiOH (metric ton) & 0 & 0 & 0.276 & 0 \\
Sulfur (metric ton) & 0 & 0 & 0 & -0.2 \\
Biomass (metric ton) & 2.719 & 2.719 & 0 & 0 \\
H2SO4 (metric ton) & $2 \mathrm{E}-04$ & $2 \mathrm{E}-04$ & 0 & 0 \\
water (metric ton) & 30.58 & 30.58 & -1.21 & 0 \\
Wastewater (metric ton) & 0.648 & 0.648 & 0 & 0 \\
Ethyl Acetate (metric ton) & $9 \mathrm{E}-04$ & $9 \mathrm{E}-04$ & 0 & 0 \\
AlCl3 (metric ton) & 0.009 & 0.009 & 0 & 0 \\
H2 (metric ton) & 0.033 & 0.033 & 0 & 0 \\
Heptane (metric ton) & 0.005 & 0.005 & 0 & 0 \\
Ethylene (metric ton) & 0.153 & 0.153 & 0 & 0 \\
Transport (tkm) & 271.9 & 271.9 & 0 & 0 \\
Electricity (GJ) & -3 & -3 & 0 & 0 \\
Hot Oil (GJ) & 0.614 & 0.614 & 0 & 0 \\
MP Steam (GJ) & 4.95 & 4.95 & 0 & 0 \\
\hline
\end{tabular}




\section{3) Summary of Unit Prices}

The USEEIO model is based on the prices of 2013 in the US, thus the prices of chemicals from the literature at different time is converted to those in 2013 by the ICIS Petrochemical Index (IPEX). ${ }^{1}$

Table S4. Unit prices for products, raw materials, and utilities in the CA process.

\begin{tabular}{|c|c|}
\hline Chemical/Utility & Unit prices (\$/unit) \\
\hline Furfural (metric ton) $)^{2}$ & 953.54 \\
\hline$p$-Xylene (metric ton $)^{2}$ & 1158.55 \\
\hline Biomass (metric ton) $)^{2}$ & 57.21 \\
\hline DME (metric ton) ${ }^{2}$ & 953.54 \\
\hline $\mathrm{H}_{3} \mathrm{PO}_{4}$ (metric ton $)^{2}$ & 572.12 \\
\hline $\mathrm{H}_{2} \mathrm{SO}_{4}$ (metric ton) ${ }^{2}$ & 238.38 \\
\hline Lime (metric ton) $)^{2}$ & 71.52 \\
\hline Gypsum (metric ton) ${ }^{a}$ & 0 \\
\hline water (metric ton $)^{3}$ & 0.80 \\
\hline Wastewater (metric ton) ${ }^{3}$ & 1.13 \\
\hline Ethyl Acetate (metric ton) $)^{2}$ & 867.72 \\
\hline $\mathrm{AlCl}_{3}(\text { metric ton })^{2}$ & 572.12 \\
\hline $\mathrm{H}_{2}$ (metric ton) ${ }^{4}$ & 1570.00 \\
\hline Heptane (metric ton) $)^{2}$ & 1430.30 \\
\hline Ethylene (metric ton) $)^{2}$ & 1239.60 \\
\hline Transport $(\mathrm{tkm})^{5}$ & 0.24 \\
\hline Electricity (GJ) ${ }^{b}$ & 17.08 \\
\hline Hot Oil (GJ) $^{c}$ & 11.41 \\
\hline Refrigerant cooling $(\mathrm{GJ})^{6}$ & 7.89 \\
\hline
\end{tabular}

${ }^{a}$ waste to be dumped; assumed to be of no value

${ }^{b}$ from Aspen Economic Analyzer and converted to the price in 2013

${ }^{c}$ Estimated from the combustion efficiency and natural gas price ${ }^{7}$ 
Table S5. Unit prices for products, raw materials, and utilities in the MSH process.

\begin{tabular}{|c|c|}
\hline Chemical/Utility & Unit prices (\$/unit) \\
\hline Furfural (metric ton) ${ }^{2}$ & 953.54 \\
\hline$p$-Xylene (metric ton $)^{2}$ & 724.69 \\
\hline $\mathrm{LiBr}$ (metric ton $)^{2}$ & 1334.95 \\
\hline $\mathrm{HBr}$ (metric ton) ${ }^{8}$ & 2708.33 \\
\hline $\mathrm{H}_{2} \mathrm{~S}$ (metric ton) ${ }^{a}$ & 0 \\
\hline $\mathrm{Br}_{2}$ (metric ton $)^{9}$ & 1847.00 \\
\hline $\mathrm{LiOH}_{(\text {metric ton })^{a}}$ & 0 \\
\hline Sulfur (metric ton) $)^{10}$ & 133.16 \\
\hline Biomass (metric ton) ${ }^{2}$ & 57.21 \\
\hline $\mathrm{H}_{2} \mathrm{SO}_{4}$ (metric ton $)^{2}$ & 238.38 \\
\hline water (metric ton) ${ }^{3}$ & 0.80 \\
\hline Wastewater (metric ton $)^{3}$ & 1.13 \\
\hline Ethyl Acetate (metric ton $)^{2}$ & 867.72 \\
\hline $\mathrm{AlCl}_{3}$ (metric ton $)^{2}$ & 572.12 \\
\hline $\mathrm{H}_{2}(\text { metric ton })^{4}$ & 1570.00 \\
\hline Heptane (metric ton) ${ }^{2}$ & 1430.30 \\
\hline Ethylene (metric ton) ${ }^{2}$ & 1239.60 \\
\hline Transport $(\mathrm{tkm})^{5}$ & 0.24 \\
\hline Electricity (GJ) ${ }^{b}$ & 17.08 \\
\hline Hot Oil $(\mathrm{GJ})^{c}$ & 11.41 \\
\hline MP Steam $(G J)^{c}$ & 11.41 \\
\hline
\end{tabular}

\footnotetext{
${ }^{a}$ Uncertainty in the price is too high, hybridization is not performed on this chemical

${ }^{b}$ from Aspen Economic Analyzer and converted to the price in 2013

${ }^{c}$ Estimated from the combustion efficiency and natural gas price ${ }^{7}$
}

\section{4) EIO-LCA: $\mathrm{CO}_{2}$ Emission During Natural Gas Combustion}

Since there is no sector in the EIO-LCA that represents the heat or steam generation, the natural gas is chosen as the fuel to produce the heat. In theory, this will cover the cradle-togate emission before the natural gas is burnt. The following step is necessary to compensate for the burning stage. 
The heating value of natural gas is taken as $23,726 \mathrm{Btu} / \mathrm{lb}(55 \mathrm{MJ} / \mathrm{kg}) .{ }^{11} \mathrm{With}$ an efficiency of 0.74 (used in Ecoinvent 3.3 database), the $\mathrm{CH}_{4}$ usage per $\mathrm{GJ}$ is:

$$
\frac{1000 \mathrm{MJ} / \mathrm{GJ}}{55 \mathrm{MJ} / \mathrm{GJ} \times 0.74}=24.57 \mathrm{kgCH} / \mathrm{GJ}
$$

The resulting $\mathrm{CO}_{2}$ emission when considering full combustion:

$$
24.57 \mathrm{kgCH}_{4} / \mathrm{GJ} \times \frac{44 \mathrm{kgCO}}{16 \mathrm{kgCH}}=67.57 \mathrm{~kg} \mathrm{CO}_{2} / \mathrm{GJ}
$$

\section{5) Technoeconomic Analysis Results Used in the Tiered Hybrid LCA}

The technoeconomic analysis results are taken from Athaley et al. ${ }^{2}$ The service-related costs are then matched to the sectors in the USEEIO.

Table S6. Operational costs for CA process. [Adapted from Athaley et al. ${ }^{2}$ Reproduced with permission from Elsevier Ltd.]

\begin{tabular}{|c|c|l|r|}
\hline Item & $\begin{array}{c}\text { Cost per year } \\
\text { (Million \$) }\end{array}$ & Sector in USEEIO & $\begin{array}{l}\text { Sector } \\
\text { number }\end{array}$ \\
\hline Total Catalyst Cost & 0.04 & $331490 /$ other secondary nonferrous metal products/us & 140 \\
\hline $\begin{array}{c}\text { Total Raw Materials } \\
\text { Cost }\end{array}$ & 75.7 & & 334 \\
\hline Total Utilities Cost & 3.7 & & 333 \\
\hline Operating Labor Cost & 1.6 & $561300 /$ employment services/us & 331 \\
\hline Maintenance Cost & 3 & $561200 /$ facilities support/us & 339 \\
\hline Operating Charges & 0.4 & $550000 /$ company and enterprise management/us & 332 \\
\hline Plant Overhead & 2.3 & $561900 /$ other support services/us & \\
\hline $\begin{array}{c}\text { General and } \\
\text { Administrative Cost }\end{array}$ & 6.75 & $561100 /$ office administration/us & \\
\hline Total Operating Cost & $\mathbf{9 3 . 5}$ & & \\
\hline
\end{tabular}

Table S7. Operational costs for MSH process. [Adapted from Athaley et al. ${ }^{2}$ Reproduced with permission from Elsevier Ltd.]

\begin{tabular}{|c|c|l|r|}
\hline Item & $\begin{array}{c}\text { Cost per year } \\
\text { (Million \$) }\end{array}$ & Sector in USEEIO & $\begin{array}{l}\text { Sector } \\
\text { number }\end{array}$ \\
\hline Total Catalyst Cost & 0.05 & $331490 /$ other secondary nonferrous metal products/us & 140 \\
\hline $\begin{array}{c}\text { Total Raw Materials } \\
\text { Cost }\end{array}$ & 79.65 & & \\
\hline Total Utilities Cost & 3.3 & & 334 \\
\hline Operating Labor Cost & 1.6 & $561300 /$ employment services/us & 333 \\
\hline Maintenance Cost & 3 & $561200 /$ facilities support/us & 331 \\
\hline Operating Charges & 0.4 & $550000 /$ company and enterprise management/us & 339 \\
\hline Plant Overhead & 2.3 & $561900 /$ other support services/us & 332 \\
\hline $\begin{array}{c}\text { General and } \\
\text { Administrative Cost }\end{array}$ & 6.75 & $561100 /$ office administration/us & \\
\hline Total Operating Cost & $\mathbf{9 7 . 0 5}$ & & \\
\hline
\end{tabular}




\section{Reference}

1. Independent Commodity Intelligence Services (ICIS) Petrochemical Index (IPEX). https://www.icis.com/explore/services/icis-insight/ipex/ (accessed July 28, 2020).

2. Athaley, A.; Annam, P.; Saha, B.; Ierapetritou, M., Techno-economic and life cycle analysis of different types of hydrolysis process for the production of $\mathrm{p}$-Xylene. Computers \& Chemical Engineering 2019, 121, 685-695.

3. Bunch, S.; Cort, K.; Johnson, E.; Elliott, D.; Stoughton, K., Water and Wastewater Annual Price Escalation Rates for Selected Cities across the United States. Office of Energy Efficiency \& Renewable Energy 2017.

4. Kuznetsov, A.; Kumar, G.; Ardagh, M. A.; Tsapatsis, M.; Zhang, Q.; Dauenhauer, P. J., On the Economics and Process Design of Renewable Butadiene from Biomass-Derived Furfural. ACS Sustainable Chemistry \& Engineering 2020.

5. Schade, W.; Doll, C.; Maibach, M.; Peter, M.; Crespo, F.; Carvalho, D.; Caiado, G.; Conti, M.; Lilico, A.; Afraz, N., Analysis of the contribution of transport policies to the competitiveness of the EU economy and comparison with the United States. COMPETE Final Report, funded by European Commission-DG TREN. Karlsruhe, Germany 2006.

6. Turton, R.; Bailie, R. C.; Whiting, W. B.; Shaeiwitz, J. A., Analysis, synthesis and design of chemical processes. Pearson Education: 2008.

7. Advanced Manufacturing Office, US Department of Energy, Benchmark the Fuel Cost of Steam Generation. (accessed July 20, 2020).

8. Alibaba Pricing of Chemicals. http://price.alibaba.com/ (accessed July 28, 2020).

9. National Minerals Information Center, United States Geological Survey (USGS), Bromine Statistics and Information. https://www.usgs.gov/centers/nmic/bromine-statisticsand-information (accessed July 20, 2020).

10. Sesay, E. Global sulphur prices weaken further on high stocks, weak demand. https://www.icis.com/explore/resources/news/2019/07/19/10393885/global-sulphur-pricesweaken-further-on-high-stocks-weak-demand (accessed July 20, 2020).

11. U.S. Department of Energy, Alternative Fuels Data Center, Fuel Properties Comparison Results (Liquefied Natural Gas (LNG)). https://afdc.energy.gov/fuels/properties (accessed July 30, 2020). 